\title{
Citrus consumption and incident dementia in elderly Japanese: the Ohsaki Cohort 2006 Study
}

\author{
Shu Zhang*, Yasutake Tomata, Kemmyo Sugiyama, Yumi Sugawara and Ichiro Tsuji \\ Department of Health Informatics and Public Health, Graduate School of Medicine, Division of Epidemiology, Tohoku \\ University School of Public Health, Sendai 980-8575, Japan
}

(Submitted 14 October 2016 - Final revision received 3 March 2017 - Accepted 7 April 2017- First published online 19 May 2017)

\section{Abstract}

Although some experimental biological studies have indicated that citrus may have preventive effects against cognitive impairment, no cohort study has yet examined the relationship between citrus consumption and incident dementia. In a baseline survey, we collected data on daily citrus intake (categorised as $\leq 2,3-4$ times/week or almost every day) and consumption of other foods using a FFQ, and used a self-reported questionnaire to collect data on other covariates. Data on incident dementia were retrieved from the Japanese Long-term Care Insurance database. A multivariate-adjusted Cox model was used to estimate the hazard ratios (HR) and $95 \%$ CI for incident dementia according to citrus consumption. Among 13373 participants, the 5.7-year incidence of dementia was 8.6\%. In comparison with participants who consumed citrus $\leq 2$ times/week, the multivariate-adjusted HR for incident dementia among those did so 3-4 times/week and almost every day was 0.92 (95\% CI $0.80,1.07)$ and $0.86(95 \%$ CI $0.73,1.01)$, respectively $\left(P_{\text {trend }}=0.065\right)$. The inverse association persisted after excluding participants whose dementia events had occurred in the first 2 years of follow-up. The multivariate HR was 1.00 (reference) for $\leq 2$ times/week, $0 \cdot 82$ (95\% CI 0.69, 0.98) for 3-4 times/week and 0.77 (95\% CI 0.64, 0.93) for almost every day $\left(P_{\text {trend }}=0.006\right)$. The present findings suggest that frequent citrus consumption was associated with a lower risk of incident dementia, even after adjustment for possible confounding factors.

Key words: Citrus: Dementia: Cohort studies: Elderly: Japan

Dementia is a major cause of disability worldwide ${ }^{(1)}$. In the absence of curative treatment, strategies for prevention of dementia are important. Since diet could be an important modifiable risk factor for dementia, nutritional interventions might help to decrease the incidence of dementia ${ }^{(2)}$.

Citrus fruits are consumed worldwide. The edible parts of citrus, such as the segment epidermis tissue and juice vesicle tissue, are rich in citrus flavonoids ${ }^{(3)}$. Previous in vivo and in vitro studies have reported that citrus flavonoids have antioxidant and anti-inflammatory bioactivities ${ }^{(4,5)}$, and can increase neuronal signalling ${ }^{(6)}$, improve metabolic functions ${ }^{(7,8)}$ and cross the blood-brain barrier ${ }^{(9)}$. In addition, some flavonoids — such as naringin and hesperidin — are essentially unique to citrus $^{(10,11)}$. Thus, it is expected that habitual intake of citrus would lower the risk of incident dementia through neuroprotective effects and promotion of brain function.

To date, only one epidemiological (cross-sectional) study has suggested that high intake of citrus ${ }^{(12)}$ was positively associated with better cognitive function (including episodic memory, executive function, perceptual speed and executive function, visuospatial skills and global cognition). Thus, an assumption that a high intake of citrus might have a preventive effect against dementia could be reasonable. However, as cross-sectional studies may be generally affected by reverse causation, any association between citrus intake and onset of dementia would be better investigated by a cohort study. To our knowledge, however, no cohort study has yet examined this issue.

The purpose of the present cohort study was to examine the relationship between habitual citrus consumption and incident dementia.

\section{Methods}

\section{Study cohort}

The design of the Ohsaki Cohort 2006 Study has been described in detail elsewhere ${ }^{(13)}$. In brief, the source population for the baseline survey comprised 31694 men and women aged $\geq 65$ years who were living in Ohsaki City, northeastern Japan, on 1 December 2006.

The baseline survey was conducted between 1 December and 15 December 2006, and follow-up of the participants started from 1 April 2007. A questionnaire was distributed by the heads of individual administrative districts to individual households and then collected by mail. In this analysis, 23091 persons who provided valid responses formed the study cohort (Fig. 1). We excluded 6333 persons who did not provide written

\footnotetext{
Abbreviations: HR, hazard ratio; LTCI, Long-term Care Insurance.
}

* Corresponding author: S. Zhang, fax +81 22717 8125, email zhangshu@med.tohoku.ac.jp 


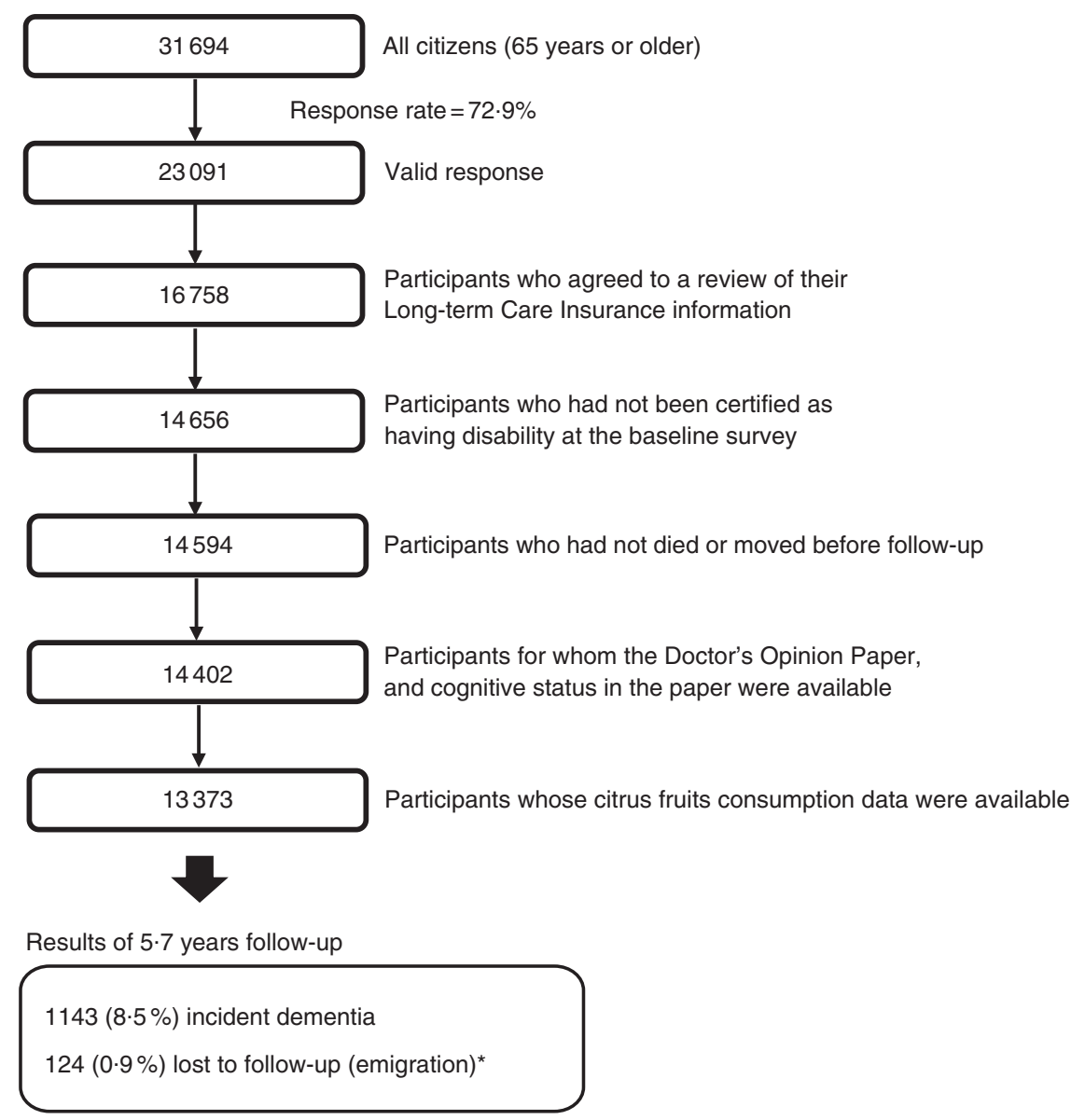

Fig. 1. Flow chart of the study participants: the Ohsaki Cohort 2006 Study. ${ }^{*}$ Without developing incident disability.

consent for review of their Long-term Care Insurance (LTCI) information, 2102 persons who had already been certified as having disability by the LTCI before follow-up (1 April 2007), sixty-two persons who had died or moved out of the district during the period of the baseline survey, 192 persons whose Doctor's Opinion Paper and cognitive status in the paper were unavailable and 1029 persons whose citrus consumption data were missing. Thus, 13373 responses were analysed for the purposes of this study. During the 5.7-year period, only 124 persons were lost to follow-up because of migration from the study area, without developing incident dementia, which provided a follow-up rate of $99.1 \%$. Among 66338 person-years, incident dementia was determined for 1143 persons (8.5\%).

\section{Consumption of citrus and other foods}

We asked about the consumption of citrus and other food items using a FFQ. The questionnaire included thirty-nine food items and several beverages. The term 'total vegetables' was defined as the sum of five food items (i.e. green vegetables, carrot and pumpkin, tomato, cabbage and lettuce and Chinese cabbage), and the term 'other fruits' referred to fruits (except citrus fruits) and fresh juice. For the majority of food items, five frequency categories were applied (almost never, 1-2 times/month, 1-2 times/week, 3-4 times/week, almost every day).
We conducted a validation study of the FFQ in which 113 respondents provided four 3 -d food records within 1 year and subsequently responded to the questionnaire. The Spearman rank correlation coefficient between citrus consumption according to the questionnaire and that according to the food records was 0.56 for men and 0.51 for women; the correlation between the consumptions measured by the two questionnaires administered 1 year apart was 0.61 for men and 0.53 for women $^{(14)}$.

\section{Covariates}

BMI was calculated as the self-reported body weight $(\mathrm{kg})$ divided by the square of the self-reported body height $(\mathrm{m})$.

The K6 was used as an indicator of psychological distress ${ }^{(15,16)}$. Using six questions, respondents were asked about their mental status over the last month. Total point scores ranged from 0 to 24 . As the optimal cut-off point for mental illness in the validation study, we classified individuals with scores of $\geq 13$ as having psychological distress ${ }^{(16)}$.

The Kihon Checklist was developed by the Ministry of Health, Labour, and Welfare of Japan to predict functional decline in community-dwelling elderly. With regard to the motor function score in the Kihon Checklist, respondents were asked about their current motor function status using five binary 
questions, yielding total point scores ranging from 0 to 5 . As the optimal cut-off point for functional decline suggested in the validation study, we classified individuals with scores of $<3$ as having better motor function ${ }^{(17)}$. With regard to the cognitive function score in the Kihon Checklist, respondents were asked about their current cognitive function status using three binary questions yielding total point scores ranging from 0 to 3 . The validity of the cognitive function score in the Kihon Checklist had been confirmed in a previous study using the Clinical Dementia Rating as a gold standard ${ }^{(18)}$.

\section{Follow-up (incident dementia)}

The primary outcome was incident dementia, defined as disabling dementia according to the criteria of the LTCI system used in $\operatorname{Japan}^{(19)}$.

The LTCI is a mandatory form of national social insurance to assist daily activity in the disabled elderly ${ }^{(20-22)}$. Everyone aged $\geq 40$ years pays premiums, and everyone aged $\geq 65$ years is eligible for formal caregiving services under a uniform standard of disability certification. The procedure for disability certification comprises two parts: (1) assessment of the degree of functional disability using a questionnaire developed by the Ministry of Health, Labour, and Welfare, and (2) reference to the Doctor's Opinion Paper prepared by the attending physician ${ }^{(23)}$.

Disabling dementia was defined as incident functional disability due to dementia according to the LTCI system, the dementia exceeding rank I (rank $\geq$ II) on the Dementia Scale (Degree of Independence in Daily Living for Elderly with Dementia), as entered on the Doctor's Opinion Paper. The Dementia Scale is classified into six ranks (0, I-IV, M. Rank M means that an individual has severe dementia-related behavioural disturbance that requires medical intervention), and a rank exceeding I is usually used as an outcome measure of incident dementia because individuals who have mild or moderate dementia are classified as rank $\mathrm{II}^{(19,24-26)}$. A previous study has shown that the Dementia Scale is well correlated with the Mini Mental State Examination score (Spearman's rank correlation coefficient $=-0.736)^{(27)}$.

We obtained a data set that included information on LTCI certification, death or emigration from Ohsaki City. All data were transferred from the Ohsaki City Government under an agreement related to Epidemiologic Research and Privacy Protection.

\section{Ethical issues}

We considered the return of completed questionnaires to imply consent to participate in the study involving the baseline survey data and subsequent follow-up of death and emigration. We also confirmed information regarding LTCI certification status after obtaining written consent along with the questionnaires returned from the subjects at the time of the baseline survey. The Ethics Committee of Tohoku University Graduate School of Medicine (Sendai, Japan) reviewed and approved the study protocol.

\section{Statistical analysis}

We counted the person-years of follow-up for each subject from 1 April 2007 until the date of incident dementia, date of emigration from Ohsaki City, date of death, incident functional disability without dementia or the end of the study period (30 November 2012), whichever occurred first.

In the present study, only $3.8 \%$ of participants almost never consumed citrus fruits, and $11 \cdot 6 \%$ consumed them 1-2 times/ month. Therefore, data for these two options were merged with the option '1-2 times/week'. Thus, citrus fruit consumption was categorised into three groups: $\leq 2,3-4$ times/week and almost every day.

The multiple adjusted Cox proportional hazards model was used to calculate the hazard ratios (HR) and 95\% CI for incident dementia according citrus consumption. Dummy variables were created for the citrus consumption groups, and respondents who consumed citrus $\leq 2$ times/week (lowest) were defined as a reference category. Multivariate models were adjusted for the following variables. Model 1 was adjusted for age $(65-69,70-74,75-79,80-84$ or $\geq 85$ years) and sex. To examine whether the association between citrus consumption and dementia was attributable to a healthy physical status or other lifestyle factors, model 2 was further adjusted for BMI $\left(<18.5,18.5-25, \geq 25 \mathrm{~kg} / \mathrm{m}^{2}\right.$ or missing), history of disease (stroke, hypertension, myocardial infarction or diabetes) (yes, no; for each term), education level (age at last school graduation: $<16,16-18, \geq 19$ years or missing), smoking (never, former, current or missing), alcohol drinking (never/former, current or missing), time spent walking $(<1, \geq 1 \mathrm{~h} /$ day or missing) and psychological distress score ( $<13, \geq 13$ or missing). In order to adjust for the influence of other dietary factors, model 3 added the consumption volume of total vegetables, and other fruits, intake of energy and protein (sex-specific tertile categories, or missing).

Considering possible reverse causality, we analysed whether the association would change if only individuals who had higher cognitive function at the baseline were selected. In this sensitivity analysis, "Cognitive function score in the Kihon Checklist $=0$ point' was defined as higher cognitive function. Another sensitivity analysis was also conducted by excluding participants whose disability event occurred in the first 2 years of follow-up.

In addition, tests of interaction were performed to investigate whether there was any difference in the relationship between citrus consumption and incident dementia in terms of sex, age ( $<75$ or $\geq 75$ years), consumption volume of total vegetables and other fruits ( $\geq$ median for both, or otherwise), and chronic conditions (stroke, hypertension, myocardial infarction or diabetes; having any one or more of these conditions, or having none of them).

All analyses above were performed by using SAS version 9.4 (SAS Inc.).

Finally, multiple imputations were conducted for missing values of all confounding factors in model 3. Based on age, sex and other observed values of confounding factors, missing values for each confounding factor were substituted with the most likely value by the multiple imputation procedure, and ten output data sets were created. Then, the Cox model was applied for the imputed data with confounding factors (without missing values) in model 3 to calculate the pooled $\mathrm{HR}$ and $95 \% \mathrm{CI}$ for incident dementia according to citrus 
consumption. Both the multiple data imputation and the Cox model were conducted using IBM SPSS Statistics 24.0 (IBM Software Group).

All statistical tests described here were two-sided, and differences at $P<0.05$ were accepted as significant.

\section{Results}

\section{Baseline characteristics}

The baseline characteristics of the 13373 participants according to citrus consumption categories are shown in Table 1 . Subjects with a higher citrus consumption frequency were less likely to be men, to have a history of stroke, to have been $<16$ years old upon completion of education, to be current smokers and alcohol drinkers, and to have psychological distress. Subjects with a lower citrus consumption frequency were less likely to consume vegetables and other fruits, and to intake protein and energy.

\section{Citrus consumption and incident dementia}

The association between citrus consumption and incident dementia is shown in Table 2. In comparison with participants who consumed citrus $\leq 2$ times/week, the incident dementia HR was 0.79 (95\% CI $0.69,0.91)$ for participants who consumed citrus 3-4 times/week and 0.77 (95\% CI $0.67,0.89)$ for those who consumed citrus almost every day $\left(P_{\text {trend }}<0.001\right.$ in the crude model). Even after adjustment for most confounding factors, the results for citrus consumption did not change substantially in model 2 (multivariate HR 1.00 (reference) for $\leq 2$ times/week, 0.87 (95\% CI 0.76, 1.00) for 3-4 times/week and 0.79 (95\% CI $0.68,0.91)$ for almost every day; $\left.P_{\text {trend }}=0.001\right)$. However, the results in model 3 became marginally non-significant.

To consider the possibility that cognitive function at the baseline might affect the association between citrus consumption and incident dementia, we analysed the association after selecting 8283 participants who had better cognitive function (cognitive function score of the Kihon Checklist $=0$ ), as shown in Table 3. Although the point HR for higher citrus consumption in all models still lower than the reference group, results of models 3 and 4 were not significant.

To examine possible reverse causality in that participants who had worse health status at the baseline might have consumed less citrus, we also analysed the association after excluding 321 participants who developed incident dementia in the first 2 years of follow-up (Table 4), but the results for citrus consumption did not change substantially; the multivariate HR was 1.00 (reference) for $\leq 2$ times/week, 0.82 (95\% CI 0.69, 0.98)

Table 1. Characteristics of participants by citrus consumption ( $n$ 13373)

(Mean values and standard deviations; percentages)

\begin{tabular}{|c|c|c|c|c|c|c|c|}
\hline & \multicolumn{6}{|c|}{ Citrus consumption } & \multirow[b]{3}{*}{$P^{*}$} \\
\hline & \multicolumn{2}{|c|}{$\leq 2$ times/week } & \multicolumn{2}{|c|}{ 3-4 times/week } & \multicolumn{2}{|c|}{ Almost every day } & \\
\hline & Mean & SD & Mean & SD & Mean & SD & \\
\hline No. of all participants & \multicolumn{2}{|c|}{5161} & \multicolumn{2}{|c|}{4041} & \multicolumn{2}{|c|}{4173} & \\
\hline Age (years) & $73 \cdot 7$ & $6 \cdot 1$ & $73 \cdot 5$ & $5 \cdot 7$ & $73 \cdot 9$ & $5 \cdot 7$ & $<0.001$ \\
\hline Sex (males, \%) & \multicolumn{2}{|c|}{57.6} & \multicolumn{2}{|c|}{43.8} & \multicolumn{2}{|c|}{$29 \cdot 8$} & $<0.001$ \\
\hline BMI $\left(k g / m^{2}\right)$ & $23 \cdot 4$ & 3.4 & $23 \cdot 6$ & $3 \cdot 3$ & $23 \cdot 8$ & $3 \cdot 3$ & $<0.001$ \\
\hline Missing (\%) & \multicolumn{2}{|c|}{$14 \cdot 7$} & \multicolumn{2}{|c|}{$11 \cdot 7$} & \multicolumn{2}{|c|}{11.8} & \\
\hline \multicolumn{8}{|l|}{ Past history (\%) } \\
\hline Stroke & \multicolumn{2}{|c|}{$3 \cdot 2$} & \multicolumn{2}{|c|}{2.7} & \multicolumn{2}{|c|}{$2 \cdot 2$} & 0.013 \\
\hline Hypertension & \multicolumn{2}{|c|}{$42 \cdot 6$} & \multicolumn{2}{|c|}{$43 \cdot 1$} & \multicolumn{2}{|c|}{44.9} & 0.063 \\
\hline Myocardial infarction & \multicolumn{2}{|c|}{5.5} & \multicolumn{2}{|c|}{$4 \cdot 4$} & \multicolumn{2}{|c|}{4.5} & 0.016 \\
\hline Diabetes & \multicolumn{2}{|c|}{$12 \cdot 5$} & \multicolumn{2}{|c|}{$11 \cdot 6$} & \multicolumn{2}{|c|}{$11 \cdot 1$} & 0.100 \\
\hline Educational level $<16$ years (missing) $(\%) \dagger$ & 31.6 & $5 \cdot 4$ & $27 \cdot 2$ & $4 \cdot 7$ & $23 \cdot 0$ & 4.5 & $<0.001$ \\
\hline Current smoker (missing) (\%) & $17 \cdot 4$ & $10 \cdot 4$ & $10 \cdot 1$ & $11 \cdot 3$ & $6 \cdot 2$ & $14 \cdot 1$ & $<0.001$ \\
\hline Current alcohol drinker (missing) (\%) & $42 \cdot 1$ & $8 \cdot 5$ & 34.5 & $9 \cdot 8$ & $24 \cdot 6$ & $11 \cdot 7$ & $<0.001$ \\
\hline Psychological distress (missing) $(\%) \ddagger$ & 4.7 & $13 \cdot 0$ & 3.6 & $12 \cdot 1$ & $3 \cdot 1$ & $14 \cdot 2$ & $<0.001$ \\
\hline Time spent walking $\geq 1 \mathrm{~h} / \mathrm{d}$ (missing) (\%) & $26 \cdot 5$ & $2 \cdot 3$ & $28 \cdot 1$ & $2 \cdot 2$ & $26 \cdot 5$ & 2.5 & 0.166 \\
\hline Better motor function (missing) $(\%) \S$ & $74 \cdot 8$ & $5 \cdot 6$ & $76 \cdot 1$ & 5.5 & $73 \cdot 5$ & $5 \cdot 7$ & 0.020 \\
\hline \multicolumn{8}{|l|}{ Intake } \\
\hline Total vegetables $(\mathrm{g} / \mathrm{d})$ & $71 \cdot 6$ & $41 \cdot 7$ & $98 \cdot 0$ & 41.9 & $120 \cdot 2$ & $45 \cdot 5$ & $<0.001$ \\
\hline Missing (\%) & \multicolumn{2}{|c|}{$14 \cdot 1$} & \multicolumn{2}{|c|}{12.3} & \multicolumn{2}{|c|}{13.5} & \\
\hline Other fruits $(g / d)$ & $59 \cdot 0$ & $57 \cdot 6$ & $97 \cdot 1$ & $62 \cdot 4$ & $132 \cdot 6$ & $76 \cdot 7$ & $<0.001$ \\
\hline Missing (\%) & & & & & & & \\
\hline Protein $(\mathrm{g} / \mathrm{d})$ & 48.5 & $14 \cdot 1$ & $55 \cdot 1$ & $13 \cdot 5$ & $58 \cdot 4$ & $13 \cdot 3$ & $<0.001$ \\
\hline Missing (\%) & & & & & & & \\
\hline Energy $(\mathrm{kJ} / \mathrm{d})$ & $5643 \cdot 1$ & $1711 \cdot 3$ & $6172 \cdot 8$ & $1673 \cdot 6$ & $6349 \cdot 8$ & $1521 \cdot 3$ & $<0.001$ \\
\hline Energy (kcal/d) & $1348 \cdot 7$ & $409 \cdot 0$ & $1475 \cdot 3$ & $400 \cdot 0$ & $1517 \cdot 6$ & 363.6 & $<0.001$ \\
\hline Missing (\%) & & & & & & & \\
\hline
\end{tabular}

* Obtained by using $x^{2}$ test for variables of proportion and one-factor ANOVA for continuous variables (missing value excluded).

$\dagger$ Age at last school graduation $<16$ years.

$\ddagger$ Kessler six-item psychological distress scale score $\geq 13$

$\S$ Motor function score of the Kihon Checklist $<3$. 
Table 2. Relationships between citrus consumption and incident dementia ( $n$ 13373)* (Adjusted hazard ratios (HR) and $95 \%$ confidence intervals)

\begin{tabular}{|c|c|c|c|c|c|c|}
\hline & \multicolumn{5}{|c|}{ Citrus consumption } & \multirow[b]{3}{*}{$P_{\text {for trend }}$} \\
\hline & \multirow[b]{2}{*}{$\leq 2$ times/week } & \multicolumn{2}{|c|}{ 3-4 times/week } & \multicolumn{2}{|c|}{ Almost every day } & \\
\hline & & $\mathrm{HR}$ & $95 \% \mathrm{Cl}$ & $\mathrm{HR}$ & $95 \% \mathrm{Cl}$ & \\
\hline No. of all participants & 5161 & \multicolumn{2}{|c|}{4041} & \multicolumn{2}{|c|}{4171} & \\
\hline Person-years & 25147 & \multicolumn{2}{|c|}{20244} & \multicolumn{2}{|c|}{20948} & \\
\hline Incident dementia (\%) & 9.7 & \multicolumn{2}{|c|}{7.9} & \multicolumn{2}{|c|}{$7 \cdot 7$} & \\
\hline Crude & Ref. & 0.79 & $0.69,0.91$ & 0.77 & $0.67,0.89$ & $<0.001$ \\
\hline Model $1 \dagger$ & Ref. & 0.82 & $0.71,0.94$ & 0.73 & $0.63,0.84$ & $<0.001$ \\
\hline Model 2‡ & Ref. & 0.87 & $0.76,1.00$ & 0.79 & $0.68,0.91$ & 0.001 \\
\hline Model $3 \S$ & Ref. & 0.92 & $0.80,1.07$ & 0.86 & $0.73,1.01$ & 0.065 \\
\hline
\end{tabular}

Ref., referent values.

* Analysis by Cox proportional hazards model.

† Model 1 was adjusted for age (65-69, 70-74, 75-79, 80-84 or $\geq 85$ years) and sex.

$\ddagger$ Model 2 was adjusted as for model 1 plus BMI $\left(<18.5,18 \cdot 5-25, \geq 25 \mathrm{~kg} / \mathrm{m}^{2}\right.$, or missing), history of disease (stroke, hypertension, myocardial infarction or diabetes (yes, no; for each term)), education level (age at last school graduation: $<16,16-18, \geq 19$ years or missing), smoking (never, former, current or missing), alcohol drinking (never/former, current or missing), time spent walking $(<1, \geq 1 \mathrm{~h} / \mathrm{d}$ or missing), psychological distress score ( $<13$, $\geq 13$ or missing).

$\S$ Model 3 was adjusted as for model 2 plus four groups of consumption volume of total vegetables, other fruits, intake of energy and protein (sex-specific tertile categories, or missing).

Table 3. Relationships between citrus consumption and incident dementia (baseline lower cognitive function excluded) $(n 8283)^{*}$ (Adjusted hazard ratios (HR) and $95 \%$ confidence intervals)

\begin{tabular}{|c|c|c|c|c|c|c|}
\hline & \multicolumn{5}{|c|}{ Citrus consumption } & \multirow[b]{3}{*}{$P_{\text {for trend }}$} \\
\hline & \multirow[b]{2}{*}{$\leq 2$ times/week } & \multicolumn{2}{|c|}{ 3-4 times/week } & \multicolumn{2}{|c|}{ Almost every day } & \\
\hline & & $\mathrm{HR}$ & $95 \% \mathrm{Cl}$ & $\mathrm{HR}$ & $95 \% \mathrm{Cl}$ & \\
\hline No. of all participants & 2975 & \multicolumn{2}{|c|}{2575} & \multicolumn{2}{|c|}{2733} & \\
\hline Person-years & 15094 & \multicolumn{2}{|c|}{13419} & \multicolumn{2}{|c|}{14111} & \\
\hline Incident dementia (\%) & $6 \cdot 2$ & \multicolumn{2}{|c|}{4.9} & \multicolumn{2}{|c|}{$5 \cdot 2$} & \\
\hline Crude & Ref. & 0.76 & $0.61,0.95$ & 0.83 & $0.67,1.03$ & 0.080 \\
\hline Model 1† & Ref. & 0.76 & $0.61,0.96$ & 0.77 & $0.61,0.96$ & 0.020 \\
\hline Model $2 \ddagger$ & Ref. & 0.79 & $0.63,1.00$ & 0.83 & $0.66,1.05$ & 0.105 \\
\hline Model $3 \S$ & Ref. & 0.85 & $0.67,1.08$ & 0.90 & $0.70,1.07$ & 0.415 \\
\hline
\end{tabular}

Ref., referent values.

* Analysis by Cox proportional hazards model.

† Model 1 was adjusted for age (65-69, 70-74, 75-79, 80-84 or $\geq 85$ years) and sex.

‡ Model 2 was adjusted as for model 1 plus BMI $\left(<18.5,18.5-25, \geq 25 \mathrm{~kg} / \mathrm{m}^{2}\right.$ or missing), history of disease (stroke, hypertension, myocardial infarction or diabetes (yes, no; for each term)), education level (age at last school graduation: $<16,16-18, \geq 19$ years or missing), smoking (never, former, current or missing), alcohol drinking (never/former, current or missing), time spent walking $(<1, \geq 1 \mathrm{~h} / \mathrm{d}$ or missing), psychological distress score ( $<13$, $\geq 13$ or missing).

$\S$ Model 3 was adjusted as for model 2 plus four groups of consumption volume of total vegetables, other fruits, intake of energy and protein (sex-specific tertile categories, or missing).

for 3-4 times/week and 0.77 (95\% CI 0.64, 0.93) for almost every day $\left(P_{\text {trend }}=0.006\right.$ in model 3$)$.

\section{Stratified analysis}

We also conducted sensitivity analysis stratified for sex, age $(<75$ or $\geq 75$ years), consumption volume of total vegetables and other fruits ( $\geq$ median for both, or otherwise), and chronic conditions (stroke, hypertension, myocardial infarction or diabetes; having any one or more of these conditions, or none of them). After adjusting for multivariate factors, the inverse correlation between citrus consumption and incident dementia did not differ between subgroups (the $P_{\text {for interaction between citrus consumption and }}$ each of the confounders was: 0.45 for sex, 0.13 for age, 0.45 for consumption volume of total vegetables and other fruits and 0.08 for chronic conditions; data not shown).

\section{Analyses of multiple imputed data}

Another sensitivity analysis was also performed by using the multiple imputed data to assess the association between citrus fruits consumption and incident dementia without missing data for confounding factors. The multivariate-adjusted results remained consistent with the results of un-imputed data. The multivariate HR was 1.00 (reference) for $\leq 2$ times/week, 0.92 (95\% CI 0.79, 1.06) for 3-4 times/week and 0.86 (95\% CI 0.73, 1.03) for almost every day $\left(P_{\text {trend }}=0.090\right.$ in model 3; data not shown).

\section{Discussion}

In this cohort study, we investigated the relationship between citrus consumption and incident dementia, and observed an inverse dose-response association between the two. Overall, 
Table 4. Relationships between citrus consumption and incident dementia (2-year dementia incidence excluded) $(n 13052)^{*}$ (Adjusted hazard ratios (HR) and $95 \%$ confidence intervals)

\begin{tabular}{|c|c|c|c|c|c|c|}
\hline & \multicolumn{5}{|c|}{ Citrus consumption } & \multirow[b]{3}{*}{$P_{\text {for trend }}$} \\
\hline & \multirow[b]{2}{*}{$\leq 2$ times/week } & \multicolumn{2}{|c|}{ 3-4 times/week } & \multicolumn{2}{|c|}{ Almost every day } & \\
\hline & & $\mathrm{HR}$ & $95 \% \mathrm{Cl}$ & $\mathrm{HR}$ & $95 \% \mathrm{Cl}$ & \\
\hline No. of all participants & 5034 & \multicolumn{2}{|c|}{3941} & \multicolumn{2}{|c|}{4077} & \\
\hline Person-years & 25009 & \multirow{2}{*}{\multicolumn{2}{|c|}{$\begin{array}{c}20134 \\
5.6\end{array}$}} & \multicolumn{2}{|c|}{20849} & \\
\hline Incident dementia (\%) & 7.4 & & & \multicolumn{2}{|c|}{$5 \cdot 6$} & \\
\hline Crude & Ref. & 0.73 & $0.61,0.86$ & 0.73 & $0.62,0.86$ & $<0.001$ \\
\hline Model $1 \dagger$ & Ref. & 0.74 & $0.63,0.88$ & 0.67 & $0.57,0.80$ & $<0.001$ \\
\hline Model $2 \ddagger$ & Ref. & 0.78 & $0.66,0.92$ & 0.73 & $0.62,0.87$ & $<0.001$ \\
\hline Model $3 \S$ & Ref. & 0.82 & $0.69,0.98$ & 0.77 & $0.64,0.93$ & 0.006 \\
\hline \multicolumn{7}{|c|}{$\begin{array}{l}\text { *Analysis by Cox proportional hazards model. } \\
\text { † Model } 1 \text { was adjusted for age (65-69, } 70-74,75-79,80-84 \text { or } \geq 85 \text { years) and sex. } \\
\text { † Model } 2 \text { was adjusted as for model } 1 \text { plus BMI }\left(<18.5,18.5-25, \geq 25 \mathrm{~kg} / \mathrm{m}^{2} \text { or missing), history of disease (stroke, hypertension, myocardial infarction or }\right. \\
\text { diabetes (yes, no; for each term)), education level (age at last school graduation: }<16,16-18, \geq 19 \text { years or missing), smoking (never, former, current } \\
\text { or missing), alcohol drinking (never/former, current or missing), time spent walking }(<1, \geq 1 \mathrm{~h} / \mathrm{d} \text { or missing), psychological distress score }(<13, \geq 13 \\
\text { or missing). } \\
\text { § Model } 3 \text { was adjusted as for model } 2 \text { plus four groups of consumption volume of total vegetables, other fruits, intake of energy and protein (sex-specific } \\
\text { tertile categories, or missing). }\end{array}$} \\
\hline
\end{tabular}

our results are consistent with a previous cross-sectional study indicating potential benefits of citrus intake in terms of cognitive function $^{(12)}$. To our knowledge, this is the first cohort study to have investigated the association between citrus consumption and incident dementia in an elderly population.

Considering the possibility that individuals with weaker cognitive function might have less opportunity to consume citrus, we investigated the effects of reverse causality. However, even when we selected only individuals who had better cognitive function at the baseline, the point estimations (HR) for each of the categories were also almost the same. In addition, even after excluding individuals who developed incident dementia in the first 2 years of follow-up, the inverse association between citrus consumption and incident dementia persisted. These findings suggest that the present results are unlikely to have been attributable to reverse causality.

Even when we performed stratified analysis by sex, age, consumption volume of total vegetables and other fruits, and chronic conditions, the results for the relationship between citrus consumption and incident dementia did not change substantially. Therefore, the association between citrus consumption and incident dementia seems difficult to explain in terms of confounding factors.

In addition, in order to minimise potential bias attributed to missing values for confounding factors, analysis of multiple imputed data was performed. However, a similar inverse association was also observed after all confounding factors had been taken into consideration. Thus, the inverse association may not be ascribed to bias of analysis in which missing values were included.

In the area where the present study was conducted, mandarin oranges (such as Satsuma (Citrus unshiu)) are the most common citrus fruit consumed in daily life ${ }^{(28)}$. The segment epidermis tissue and juice vesicle tissue of these citrus fruits contain citrus flavonoids such as naringin, hesperidin, narirutin and neohesperidin ${ }^{(3)}$. Some experimental biological studies have suggested that these citrus flavonoids have neuroprotective effects (antioxidant properties, anti-inflammation and signalling regulation) ${ }^{(29-32)}$. Therefore, the inverse relationship observed in the present study might have been attributable to these citrus flavonoids.

Our study had a number of strengths: (1) it was a large population-based cohort study involving 13373 persons; (2) the follow-up rate was very high (99.1\%); (3) many confounding factors were taken into account; and (4) the study subjects lived in an area in which citrus fruits are widely consumed.

However, there were also several limitations. First, the causes of dementia were not evaluated, and therefore the mechanism responsible for reduction of incident dementia by citrus consumption remained unclarified. Second, information on citrus consumption using the FFQ was obtained only at the baseline, and the study subjects might have changed their citrus consumption during the course of follow-up. Third, potential confounding factors could not be disregarded. Particularly, it could not be ruled out that residual diet-related confounding factors were present, since our FFQ data could not fully consider diet quality. Lastly, the relatively short follow-up time of the participants might have compromised our findings. A cohort study with a longer follow-up might therefore be desirable.

In conclusion, the present study has shown that citrus consumption tended to be associated with a lower risk of incident dementia in elderly Japanese individuals. Our results suggest that habitual citrus consumption may have a preventive effect against the incident risk of dementia. Further prospective studies in other populations and settings will be necessary to confirm the association between citrus consumption and dementia.

\section{Acknowledgements}

The authors would like to thank Yoshiko Nakata, Yukiko Asano, Mao Suzuki and Mami Takahashi for their technical assistance.

This work was supported by Health Sciences Research grants (nos. H24-Choju-Ippan-005 and H26-Junkankitou (Seisaku)-Ippan001) from the Ministry of Health, Labour and Welfare of Japan. 
Sponsors play no role in the design, methods, subject recruitment, data collections, analysis and preparation of paper.

S. Z. and I. T. designed research; Y. T. and K. S. conducted research; S. Z. and Y. T. analysed data; S. Z. wrote the paper; Y. T., K. S., Y. S. and I. T. gave the constructive suggestions; S. Z. had primary responsibility for final content. All authors read and approved the final manuscript.

None of the authors has any conflicts of interest to declare.

\section{References}

1. Lefstad E (2015) The epidemiology and impact of dementia: current state and future trends. www.who.int/mental_health/ neurology/dementia/dementia_thematicbrief_epidemiology. pdf (accessed March 2016)

2. Feart C, Samieri C, Rondeau V, et al. (2009) Adherence to a Mediterranean diet, cognitive decline, and risk of dementia. JAMA 302, 638-648.

3. Nogata Y, Sakamoto K, Shiratsuchi H, et al. (2006) Flavonoid composition of fruit tissues of citrus species. Biosci Biotechnol Biochem 70, 178-192.

4. Parhiz H, Roohbakhsh A, Soltani F, et al. (2015) Antioxidant and anti-inflammatory properties of the citrus flavonoids hesperidin and hesperetin: an updated review of their molecular mechanisms and experimental models. Phytother Res 29, 323-331.

5. Alam MA, Subhan N, Rahman MM, et al. (2014) Effect of citrus flavonoids, naringin and naringenin, on metabolic syndrome and their mechanisms of action. Adv Nutr 5, 404-417.

6. Spencer JP (2007) The interactions of flavonoids within neuronal signalling pathways. Genes Nutr 2, 257-273.

7. Orhan IE (2014) Implications of some selected flavonoids towards Alzheimer's disease with the emphasis on cholinesterase inhibition and their bioproduction by metabolic engineering. Curr Pharm Biotechnol 15, 352-361.

8. Mulvihill EE \& Huff MW (2012) Protection from metabolic dysregulation, obesity, and atherosclerosis by citrus flavonoids: activation of hepatic PGC1alpha-mediated fatty acid Oxidation. PPAR Res 2012, 857142.

9. Youdim KA, Shukitt-Hale B \& Joseph JA (2004) Flavonoids and the brain: interactions at the blood-brain barrier and their physiological effects on the central nervous system. Free Radic Biol Med 37, 1683-1693.

10. Tripoli E, Guardia ML, Giammanco S, et al. (2007) Citrus flavonoids: molecular structure, biological activity and nutritional properties: a review. Food Chem 104, 466-479.

11. Rothwell JA, Perez-Jimenez J, Neveu V, et al. (2013) PhenolExplorer 3.0: a major update of the Phenol-Explorer database to incorporate data on the effects of food processing on polyphenol content. Database (Oxford) 2013, bat070.

12. Nurk E, Refsum H, Drevon CA, et al. (2010) Cognitive performance among the elderly in relation to the intake of plant foods. The Hordaland Health Study. Br J Nutr 104, 1190-1201.

13. Kuriyama S, Nakaya N, Ohmori-Matsuda K, et al. (2010) The Ohsaki Cohort 2006 Study: design of study and profile of participants at Baseline. J Epidemiol 20, 253-258.

14. Ogawa K, Tsubono Y, Nishino Y, et al. (2003) Validation of a food-frequency questionnaire for cohort studies in rural Japan. Public Health Nutr 6, 147-157.
15. Kessler RC, Andrews G, Colpe LJ, et al. (2002) Short screening scales to monitor population prevalences and trends in non-specific psychological distress. Psychol Med 32, 959-976.

16. Kessler RC, Green JG, Gruber MJ, et al. (2010) Screening for serious mental illness in the general population with the $\mathrm{K} 6$ screening scale: results from the WHO World Mental Health (WMH) survey initiative. Int J Methods Psychiatr Res 19, Suppl. 1, 4-22.

17. Tomata Y, Hozawa A, Ohmori-Matsuda K, et al. (2011) [Validation of the Kihon Checklist for predicting the risk of 1-year incident long-term care insurance certification: the Ohsaki Cohort 2006 Study]. Nihon Koshu Eisei Zasshi 58, 3-13.

18. Meguro K \& Team. KP (2012) The validity of the Basic Checklist in the old-old population: The Kurihara Project. Jpn J Geriatr Psychiat, 725-730.

19. Ikeda A, Yamagishi K, Tanigawa T, et al. (2008) Cigarette smoking and risk of disabling dementia in a Japanese rural community: a nested case-control study. Cerebrovasc Dis $\mathbf{2 5}$, 324-331.

20. Ikegami N (1997) Public long-term care insurance in Japan. JAMA 278, 1310-1314.

21. Tsutsui T \& Muramatsu N (2005) Care-needs certification in the long-term care insurance system of Japan. J Am Geriatr Soc 53, 522-527.

22. Imahashi K, Kawagoe M, Eto F, et al. (2007) Clinical status and dependency of the elderly requiring long-term care in Japan. Tohoku J Exp Med 212, 229-238.

23. Moriyama Y, Tamiya N, Kamimura A, et al. (2014) Doctors' Opinion Papers in long-term care need certification in Japan: comparision between clinic and advanced treatment hospital settings. Public Policy Admin Res 4, 31-37.

24. Yamamoto T, Kondo K, Hirai H, et al. (2012) Association between self-reported dental health status and onset of dementia: a 4-year prospective cohort study of older Japanese adults from the Aichi Gerontological Evaluation Study (AGES) Project. Psychosom Med 74, 241-248.

25. Okumura Y, Kuze J \& Higuchi K (2009) Prevalence of problematic behaviors in the ambulant elderly with dementia. Kawasaki J Med Welfare 15, 27-35.

26. Meguro K, Tanaka N, Kasai M, et al. (2012) Prevalence of dementia and dementing diseases in the old-old population in Japan: the Kurihara Project. Implications for Long-Term Care Insurance data. Psychogeriatrics 12, 226-234.

27. Hisano S (2009) The relationship between Revised Hasegawa Dementia Scale (HDS-R), Mini-Mental State Examination (MMSE) and Bed-fast Scale, Dementia Scale. Jpn J Geriatr Psychiat 20, 883-891.

28. Shimizu T (2002) Issues of supply and demand trends and agriculture of citrus. Norin Kinyu 55, 2-23.

29. Williams RJ, Spencer JP \& Rice-Evans C (2004) Flavonoids: antioxidants or signalling molecules? Free Radic Biol Med 36, 838-849.

30. Vauzour D, Vafeiadou K, Rice-Evans C, et al. (2007) Activation of pro-survival Akt and ERK1/2 signalling pathways underlie the anti-apoptotic effects of flavanones in cortical neurons. J Neurochem 103, 1355-1367.

31. Hwang SL, Shih PH \& Yen GC (2012) Neuroprotective effects of citrus flavonoids. J Agric Food Chem 60, 877-885.

32. Hwang SL, Lin JA, Shih PH, et al. (2012) Pro-cellular survival and neuroprotection of citrus flavonoid: the actions of hesperetin in PC12 cells. Food Funct 3, 1082-1090. 